\title{
OPTIMAL CONTROL OF PIEZOELECTRIC ANISOTROPIC PLATES
}

\author{
ISABEL NARRA FIGUEIREDO AND GEORG STADLER
}

\begin{abstract}
This paper is concerned with the application of optimal control techniques to a static piezoelectric anisotropic thin plate model. To take physical limitations into account, the space of electric potential fields is restricted, i.e., control constraints are imposed. After deriving the first-order necessary conditions for the problem, a primal-dual active set strategy for its solution is presented. In a numerical study we consider a plate composed of two layers of different piezoelectric materials. We investigate the abilities of our approach and the influence of certain parameters.
\end{abstract}

KEYWORDS: piezoelectric material, optimal control, laminated plate.

\section{The anisotropic plate model}

Piezoelectric materials deform when they are subject to an electric field. This property, known as the inverse piezoelectric effect can be used to design smart materials and structures, which are nowadays used in various areas such as mechatronics and micro electro mechanical systems. We refer to $[1,2]$ for a general introduction to piezoelectric materials.

In order to make these materials behave in the desired way, one can either combine a large number of simulations with physical intuition, or utilize an optimizationbased method. We apply the latter approach to a model for piezoelectric anisotropic plates derived in [3] (see also [4] for a similar model). To be precise, we formulate the problem as optimal control problem, i.e., we search for an applied electric field that generates a deformation of the plate, which is as close as possible to a given desired deformation.

The piezoelectric anisotropic plate model we use is deduced by an asymptotic expansion method from a three-dimensional model in [3]. While the threedimensional equations are coupled, in the asymptotic model, these equations become splitted up into two parts. The first one establishes that the displacement is a Kirchhoff-Love displacement, which is characterized by the solution of a linear two-dimensional partial differential equation in the middle plane of the plate. The second part gives an explicit formula for the electric potential in the whole plate. This is remarkable, since in the tree-dimensional equations the deformation

\footnotetext{
Received March 06, 2006.
} 
and the electric potential are coupled by means of a partial differential equation. The formula is given by a second order polynomial with respect to the thickness variable. Moreover, it can be shown that for a laminated plate the polynomial's coefficients depend on the transverse and tangential components of the displacement and the material coefficients.

We now summarize the asymptotic piezoelectric model equations. Let $\omega \subset \mathbb{R}^{2}$, bounded, be the middle plane of a piezoelectric thin plate with thickness $2 h, h>0$ a small constant. The plate occupies the domain $\Omega:=\omega \times(-h, h)$, and we denote by $\mathbf{u}=\left(u_{1}, u_{2}, u_{3}\right) \in V_{K L}$ the displacement of the plate. Here, $V_{K L}$ denotes the Kirchhoff-Love mechanical displacement space including the Dirichlet boundary conditions (the plate is assumed to be clamped on a part of the boundary with positive measure). The plate can be subject to volume forces in $\Omega$ and boundary tractions on $\partial \Omega$, and, more interestingly, subject to electric potentials on its upper and lower surface. Since in the asymptotic model equations only the difference between upper and lower electric potential enters, we only introduce a variable for this difference (defined on the middle plane of the plate) and denote it by $\phi \in L^{2}(\omega)$, where $L^{2}(\omega):=\left\{\varphi: \omega \rightarrow \mathbb{R}: \int_{\omega} \varphi^{2} d x<\infty\right\}$ is the space of square integrable functions. For reasons of simplicity, in the sequel we refer to $\phi$ as "electric potential", but we always mean the difference between the electric potentials applied to the upper and lower surface.

Then, the displacement $\mathbf{u}$ is characterized as the solution to the following variational problem

$$
\left\{\begin{array}{l}
\text { Find } \mathbf{u} \in V_{K L} \text { such that } \\
a(\mathbf{u}, \mathbf{v})=\left(\ell_{1}, \mathbf{v}\right)+\left(\ell_{2} \phi, \mathbf{v}\right) \text { for all } \mathbf{v} \in V_{K L} .
\end{array}\right.
$$

Above, the bilinear form $a(\cdot, \cdot)$ involves the elastic, piezoelectric and dielectric coefficients, see [3]. The linear form $\left(\ell_{1}, \cdot\right)$ collects volume and surface forces, while $\left(\ell_{2} \phi, \cdot\right)$ can be interpreted as an electric force induced by the electric potential $\phi$ (to be precise, the difference of the applied electric potentials on the upper and lower surface of the plate). Note that $\ell_{2}$ again involves elastic, piezoelectric and dielectric material parameters, and that $\phi$ enters linearly into $\ell_{2} \phi$.

It can be shown that the variational problem (1) admits a unique solution for sufficiently smooth data, in particular for each $\phi \in L^{2}(\omega)$. After having obtained the displacement $\mathbf{u}$ from (1), one can derive the electric potential in the plate from a second order polynomial (cf. [3]). 


\section{The optimal control problem}

In this section we formulate the optimal control problem, state basic properties and derive the necessary optimality conditions. To deform the piezoelectric plate in a desired way, we minimize a cost functional that involves the displacement $\mathbf{u}$ and the electic potential $\phi$. It is, for $(\mathbf{u}, \phi) \in V_{K L} \times L^{2}(\omega)$, defined by

$$
J(\mathbf{u}, \phi):=\frac{1}{2} \int_{\Omega}\left\|E\left(\mathbf{u}-\mathbf{u}_{d}\right)\right\|^{2} d x+\frac{\gamma}{2} \int_{\omega}|\phi|^{2} d x .
$$

Here, $\|\cdot\|$ denotes the Euclidean norm in $\mathbb{R}^{3}$, and $E \in \mathbb{R}^{3 \times 3}$ a diagonal matrix with nonnegative entries. The vector function $\mathbf{u}_{d}$ is a given displacement one would like to achieve by choosing an appropriate electric potential $\phi$. The parameter $0<\gamma \ll 1$ weights the control cost in (2) and makes the functional strictly convex. To implement physical limitations in our problem formulation, we restrict ourselves to the following set of admissible electric potentials:

$$
U_{a d}=\left\{\phi \in L^{2}(\omega): a(x) \leq \phi(x) \leq b(x) \text { a.e. on } \omega\right\},
$$

where $a, b \in L^{2}(\omega)$ with $a(x)<b(x)$ almost everywhere are pointwise bounds for the electric potential. Then, the optimal control problem under consideration is

minimize $J(\mathbf{u}, \phi)$ subject to the state equation (1) and $(\mathbf{u}, \phi) \in V_{K L} \times U_{a d}$. (P)

It is standard to argue that the optimal control problem $(P)$ admits a solution. Uniqueness of this solution follows from the linearity of the state equation (1) and the strict convexity of the cost functional (2).

We are now prepared to derive the first-order necessary conditions for the problem $(P)$. Therefore, we introduce the functional $e: V_{K L} \times L^{2}(\omega) \rightarrow V_{K L}^{\star}$ defined by

$$
\langle e(\mathbf{u}, \phi), \mathbf{v}\rangle:=a(\mathbf{u}, \mathbf{v})-\left(\ell_{1}, \mathbf{v}\right)-\left(\ell_{2} \phi, \mathbf{v}\right) \text { for } \mathbf{v} \in V_{K L},
$$

where $\langle\cdot, \cdot\rangle$ denotes the duality pairing between $V_{K L}^{\star}$ and $V_{K L}$. Using this notation, the weak form of the state equation (1) becomes $e(\mathbf{u}, \phi)=0$ in $V_{K L}^{\star}$. To derive the necessary optimality conditions, we use the Lagrangian functional $\mathscr{L}: V_{K L} \times$ $L^{2}(\omega) \times V_{K L} \rightarrow \mathbb{R}$ defined by

$$
\mathscr{L}(\mathbf{u}, \phi, \mathbf{p}):=J(\mathbf{u}, \phi)+\langle e(\mathbf{u}, \phi), \mathbf{p}\rangle .
$$


Since the gradient $e^{\prime}(\mathbf{u}, \phi)$ of $e(\mathbf{u}, \phi)$ is surjective for all $(\mathbf{u}, \phi) \in V_{K L} \times L^{2}(\omega)$, there exists (see, e.g., [5]), $\mathrm{p}^{\star} \in V_{K L}$ such that

$$
\begin{aligned}
& \frac{\partial}{\partial \mathbf{u}} \mathscr{L}\left(\mathbf{u}^{\star}, \phi^{\star}, \mathbf{p}^{\star}\right)=0, \\
& \frac{\partial}{\partial \mathbf{p}} \mathscr{L}\left(\mathbf{u}^{\star}, \phi^{\star}, \mathbf{p}^{\star}\right)=0, \\
& \frac{\partial}{\partial \phi} \mathscr{L}\left(\mathbf{u}^{\star}, \phi^{\star}, \mathbf{p}^{\star}\right)\left(\phi-\phi^{\star}\right) \geq 0 \text { for all } \phi \in U_{a d} .
\end{aligned}
$$

Evaluating (3), one obtains the optimality system that we summarize in the following theorem. For the proof we refer to [6], where a similar result is established.

Theorem 1. The solution $\left(\mathbf{u}^{\star}, \phi^{\star}\right)$ of $(P)$ is characterized by the existence of $\left(p^{\star}, \lambda_{a}^{\star}, \lambda_{b}^{\star}\right) \in V_{L K} \times\left(L^{2}(\omega)\right)^{2}$ such that

$$
\begin{aligned}
& a\left(\mathbf{u}^{\star}, \mathbf{v}\right)-\left(\ell_{2} \phi^{\star}, \mathbf{v}\right)=\left(\ell_{1}, \mathbf{v}\right) \text { for all } \mathbf{v} \in V_{K L}, \\
& a\left(\mathbf{v}, \mathbf{p}^{\star}\right)+\left(\mathbf{v}, E^{2} \mathbf{u}^{\star}\right)=\left(\mathbf{v}, E^{2} \mathbf{u}_{d}\right) \text { for all } \mathbf{v} \in V_{K L}, \\
& \gamma \phi^{\star}+\lambda_{b}^{\star}-\lambda_{a}^{\star}-\ell_{2}^{\star} \mathbf{p}^{\star}=0, \\
& \phi^{\star}-a \geq 0, \lambda_{a}^{\star} \geq 0,\left(\phi^{\star}-a, \lambda_{a}^{\star}\right)=0 \\
& b-\phi^{\star} \geq 0, \lambda_{b}^{\star} \geq 0,\left(b-\phi^{\star}, \lambda_{b}^{\star}\right)=0 .
\end{aligned}
$$

We remark that the equations $(4 \mathrm{~d}),(4 \mathrm{e})$ are the complementarity conditions corresponding to the inequality constraints in $U_{a d}$. In the absence of these constraints, i.e., in the case $U_{a d}=L^{2}(\omega)$, only the equations (4a), (4b) and (4c) with $\lambda_{a}^{\star}=\lambda_{b}^{\star}=0$ have to be taken into account. Hence, in this case the optimality system becomes linear.

However, in general the system (4) is nonlinear and therefore its solution requires an iterative approach. To motivate the algorithm presented in the next section we introduce

$$
\lambda^{\star}:=\lambda_{b}^{\star}-\lambda_{a}^{\star}
$$

Then, the equations (4d), (4e) can be rewritten as the following nonsmooth equation:

$$
\lambda^{\star}-\max \left(0, \lambda^{\star}+\sigma\left(\phi^{\star}-b\right)\right)-\min \left(0, \lambda^{\star}+\sigma\left(\phi^{\star}-a\right)\right)=0
$$

for arbitrary $\sigma>0$. In fact, taking into account (5), it is an easy exercise to show that (6) is equivalent to $(4 \mathrm{~d}),(4 \mathrm{e})$. 


\section{Solution algorithm for $(P)$}

We now state our algorithm for the solution of the nonlinear optimality system (4a)-(4e). The algorithm can either be seen as an active set strategy or as generalized Newton method (see [7]). It derives in each iteration $k$ estimates $\mathcal{A}_{a}^{k}$ and $\mathcal{A}_{b}^{k}$ for the active sets at the solution, i.e., for the sets where the inequality bounds in $U_{a d}$ are active meaning $\phi^{\star}=a$ or $\phi^{\star}=b$. These estimates are motivated by (7), and for a current iterate $\left(\phi^{k-1}, \lambda^{k-1}\right)$ given by

$$
\begin{aligned}
& \mathcal{A}_{a}^{k}=\left\{x \in \omega: \lambda^{k-1}+\sigma\left(\phi^{k-1}-a\right)<0\right\}, \\
& \mathcal{A}_{b}^{k}=\left\{x \in \omega: \lambda^{k-1}+\sigma\left(\phi^{k-1}-b\right)>0\right\} .
\end{aligned}
$$

The algorithm presented below only involves $\phi$ and $\lambda$, since only these variables occur in the nonlinearity of the optimality system (4). The displacement $\mathbf{u}$ depends linearly on these variables.

\section{Algorithm 1.}

(1) Initialize $\left(\phi^{0}, \lambda^{0}\right)$, choose $\sigma>0$ and set $k:=1$.

(2) Determine $\mathcal{A}_{a}^{k}$ and $\mathcal{A}_{b}^{k}$ according to (7) and derive the inactive set $\mathcal{I}^{k}:=$ $\omega \backslash\left(\mathcal{A}_{a}^{k} \cup \mathcal{A}_{b}^{k}\right)$.

(3) If $k \geq 2$ and $\mathcal{A}_{a}^{k}=\mathcal{A}_{a}^{k-1}, \mathcal{A}_{b}^{k}=\mathcal{A}_{b}^{k-1}$ stop. Else,

(4) Solve the equations (4a)-(4c) with the settings

$$
\phi^{k}:=\left\{\begin{array}{l}
a \text { on } \mathcal{A}_{a}^{k}, \\
b \text { on } \mathcal{A}_{b}^{k},
\end{array} \quad \text { and } \quad \lambda^{k}:=0 \text { on } \mathcal{I}^{k},\right.
$$

set $k:=k+1$ and go to step 2 .

We remark that the system in step 4 of Algorithm 1 is linear and admits a unique solution. The above algorithm is easy to implemented, and it uses the simple stopping criterion given in step 2 of Algorithm 1. Namely, the iteration is terminated as soon as the estimates for the active sets coincide in two consecutive iterations. Then, as can be seen easily, the last iterate is the solution of the problem.

The method can also be seen as a generalized Newton method (see [7]). This interpretation allows to give the following local convergence result for the infinitedimensional algorithm.

Theorem 2. If $\sigma=\gamma$ and the initializations $\left(\phi^{0}, \lambda^{0}\right)$ are chosen such that $\| \phi^{\star}-$ $\phi^{0} \|_{L^{2}}$ and $\left\|\lambda^{\star}-\lambda^{0}\right\|_{L^{2}}$ are sufficiently small, the iterates $\left(\phi^{k}, \lambda^{k}\right)$ of Algorithm 1 converge to $\left(\phi^{\star}, \lambda^{\star}\right)$ in $L^{2}(\omega) \times L^{2}(\omega)$ at a superlinear rate. 
Proof: The proof relies on the interpretation of Algorithm 1 as semismooth Newton method and uses the smoothing property of the control-to-state mapping, i.e., the mapping $\phi \rightarrow \mathbf{u}$. For details we refer to [7].

Note that the above results hold in the infinite-dimensional function space $L^{2}(\omega) \times$ $L^{2}(\omega)$. Clearly, an analogous convergence result holds for appropriate discretizations of the optimality system (4) as well. However, besides a possible theoretical interest in analyzing methods before discretizing them, an infinite-dimensional approach might give more insight into the problem structure and allows one to investigate, e.g., smoothing properties of the involved differential operators or mesh-independence of the algorithm.

\section{Numerical results}

The problem $(P)$ is discretized by means of finite elements on a regular rectangular mesh. To be more precise, for the tangential displacements $\left(u_{1}, u_{2}\right)$ we use Melosh finite elements (see, e.g., [8]). These elements only involve the displacements, which results into 8 shape functions for both components $u_{1}, u_{2}$ on each finite element. The discretization of the transverse displacement $u_{3}$ is based on Adini finite elements [8], which take into account the two first order derivatives. This leads to 12 shape functions for $u_{3}$ on each finite element. Our algorithm is implemented in MATLAB and uses routines from the finite element toolbox CALFEM [9].

4.1. Example 1. In this example, we consider a plate consisting of two laminas with the same thickness. They are made of two different PZT ceramic materials, whose piezoelectric, dielectric and elastic coefficients are taken from the Tables VIII and XI of [10].

The plate's middle surface $\omega$ is given by $[0,1] \times[0,3]$ (the unit used here and below is meter), its thickness is $2 \times 10^{-2}$ and it is clamped on the side $[0,1] \times\{0\}$. Our finite element discretization uses 20 times 60 elements in $x$ - and $y$-direction, respectively. In this first example, we are only interested in controlling the transverse component of the displacement. Therefore, we choose $E:=\operatorname{diag}(0,0,1)$ resulting in a cost functional $J$, in which only $u_{3}$ and $\phi$ occur. The three components of the desired state $\mathbf{u}_{d}$ are given by $u_{d, 1}=u_{d, 2}=0$, and

$$
u_{d, 3}(x, y)=2 \times 10^{-4} \cdot \begin{cases}0 & \text { for } 0 \leq y \leq 1 \\ -2(y-1)^{3}+3(y-1)^{2} & \text { for } 1 \leq y \leq 2 \\ 1 & \text { for } 2 \leq y \leq 3\end{cases}
$$




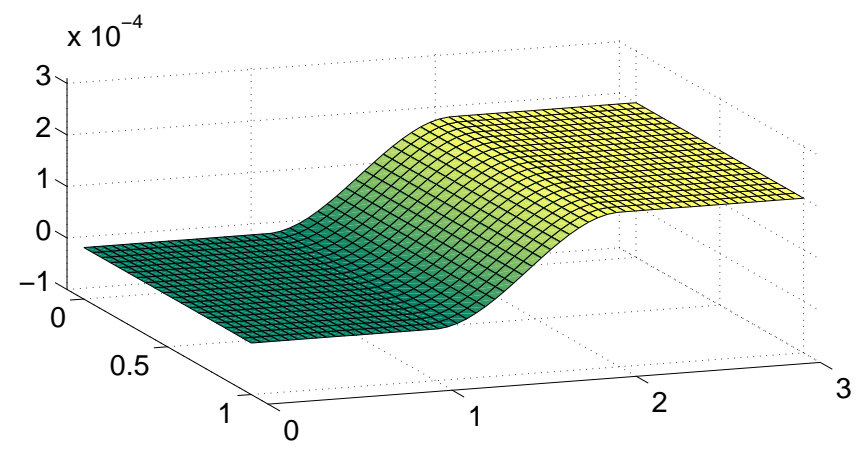

FIGURE 1. Desired displacement $\mathbf{u}_{d}$ of the middle plane.

see Figure 1. We remark, that the transverse component of the deformation can only be influenced by an electric potential since the plate consists of two different lamina. For piezoelectric plates made from only one material with constant piezoelectric, dielectric and elastic coefficients, the tangential and transverse displacement in the asymptotic model are not coupled and thus the transverse displacement is independent of the applied electric potential. Such a simplified model is considered in [11]. Since we are mainly interested in the inverse piezoelectric effect, we assume the mechnical forces to be zero, i.e., $\ell_{1}=0$ in (1).

In the optimal control problem $(P)$ we choose the control weight $\gamma=10^{-15}$. Such a small value for $\gamma$ becomes necessary due to the different scaling of the variables $\mathbf{u}$ and $\phi$, which is due to the material parameters. For the present problem, $\phi$ is of order $10^{2}$, while the displacement $\mathbf{u}$ is of order $10^{-4}$. To avoid ill-conditioned systems due to these different scales, in our numerical implementation we use rescaled variables $\tilde{\mathbf{u}}$ and $\tilde{\phi}$ instead of $\mathbf{u}$ and $\phi$.

First, we show the result obtained in the absence of control constraints, i.e., for the case $U_{a d}=L^{2}(\omega)$. The left plot in Figure 2 shows the optimal electric potential $\phi$, while the right plot shows the displacement of the middle plane obtained by the electric forces induced by $\phi$. As can be seen from Figure 2, the optimal electric field is relatively complicated, but it deforms the middle plane of the plate in the desired way.

Next, we show the results for the case that constant control constraints $a \equiv$ $-175 \mathrm{~V}$ and $b \equiv 175 \mathrm{~V}$ (here, $\mathrm{V}$ means the SI unit Volt) have to be taken into account. The resulting nonlinear optimality system is solved using Algorithm 1 with the parameter $\sigma=1$. The method detects the solution after only 5 iteration steps. Since the space of possible electric fields is restricted, the resulting displacement is slightly worse (i.e., further away from the desired state). However, 

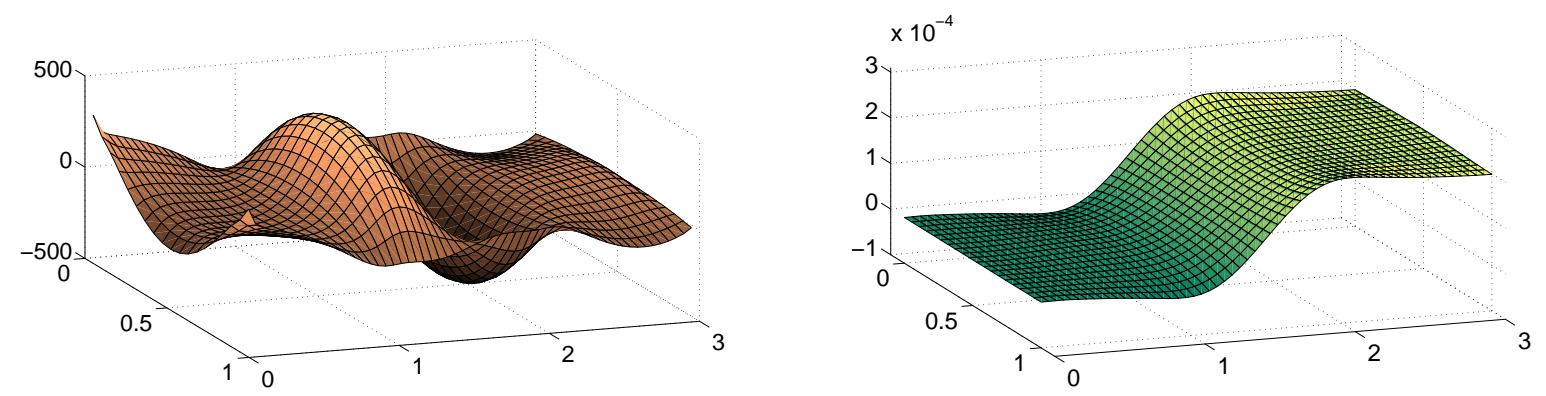

FIGURE 2. Optimal electric potential $\phi$ for the case without control constraints (left) and deformed middle plane (right).
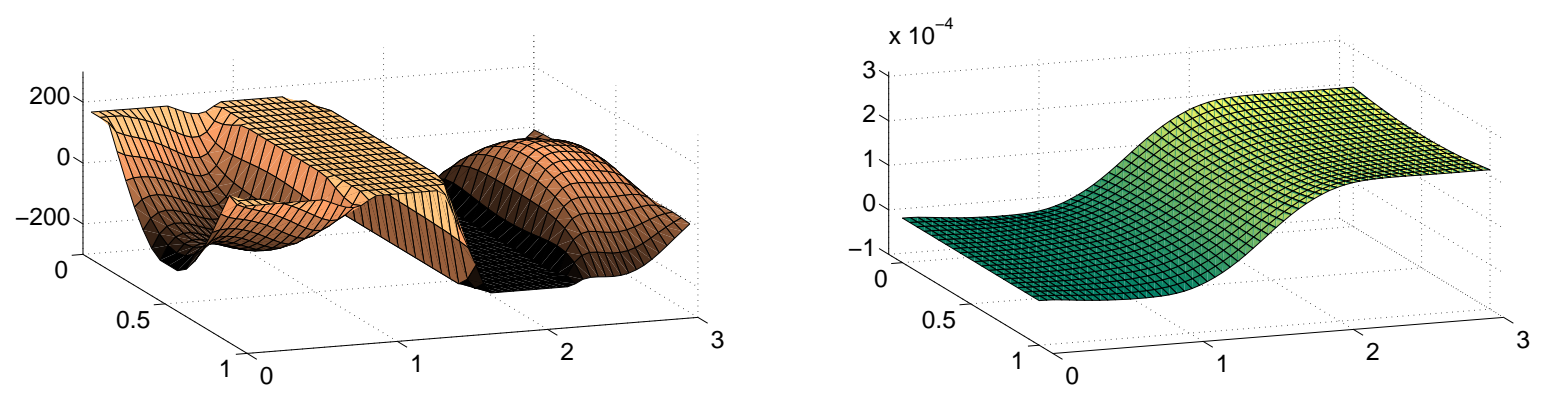

FIGURE 3. Optimal electric potential $\phi$ in the presence of the control constraints $a \equiv-175$ and $b \equiv 175$ and deformed middle plane (right).

the difference is so small that it cannot be seen from comparing the right plots of the Figures 2 and 3.

4.2.Example 2. This example uses the same geometry and data as Example 1, but a modified cost functional in order to show the flexibility of an optimization-based approach. To motivate the modification, we show in the left plot of Figure 4 a top view of the deformed middle plane of the plate that is subject to the unconstrained optimal potential shown in Figure 2. For reasons of graphical representation, the displacement has been multiplied by the factor $10^{4}$. As can be seen from Figure 4 , the electric potential does not only influence the transverse displacement in the desired way, it also affects the tangential displacement $\left(u_{1}, u_{2}\right)$. Since this might be an undesired behavior, one can try to keep the first component $u_{1}$ of the tangential displacement small by choosing $E=\operatorname{diag}(1,0,1)$ in the cost functional (2). This involves $u_{1}$ in the cost functional and acts as penalization if $u_{1} \neq 0$ (since $u_{d, 1}=0$ ). This modified choice for $E$ results in the displacement shown in the middle plot of Figure 4. For the right plot, we further increased the weight for 

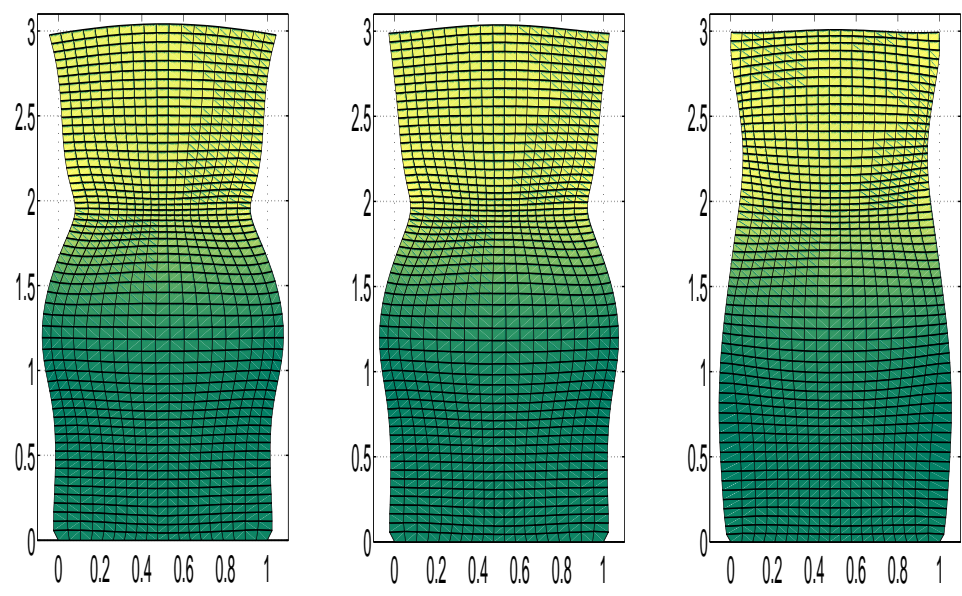

FIGURE 4. Top view of deformed plate for different choices of the matrix $E$ in the cost functional; $E=\operatorname{diag}(0,0,1)$ (left), $E=$ $\operatorname{diag}(1,0,1)$ (middle), $E=\operatorname{diag}(5,0,1)$ (right).
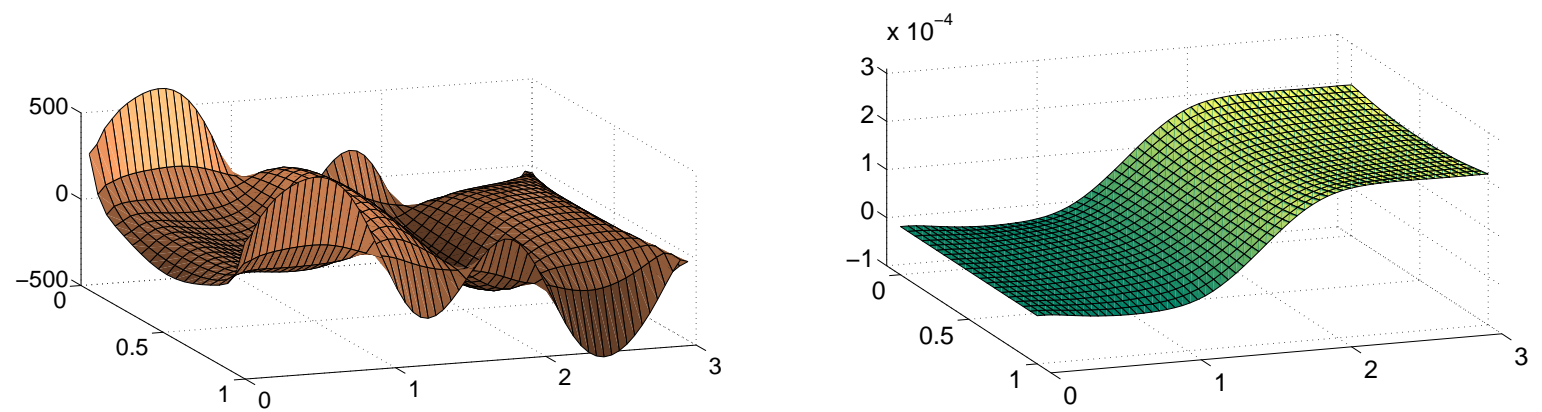

FIGURE 5. Optimal electric potential $\phi$ (left) and deformed middle plane for the choice $E=\operatorname{diag}(5,0,1)$ (right).

the first component $u_{1}$ by choosing $E=\operatorname{diag}(5,0,1)$. Now, it can clearly be seen that this leads to a smaller deformation in $x$-direction. However, the transverse displacement is still close to the desired one, as can be seen from Figure 5, where the optimal electric potential and the resulting deformation for $E=\operatorname{diag}(5,0,1)$ are shown.

\section{References}

[1] T. Ikeda. Fundamentals of Piezoelectricity. Oxford University Press, New York, 1990.

[2] H Smith. Smart material systems: model development. Frontiers in applied mathematics. SIAM, Philadelphia, 2005.

[3] I.N. Figueiredo and C. Leal. A piezoelectric anisotropic plate model. Asymptotic Analysis, 44(34):327-346, 2005. 
[4] M. Rahmoune, A. Benjeddou, and R. Ohayon. New thin piezoelectric plate models. Journal of Intelligent Material Systems and Structures, 9:1017-1029, 1998.

[5] H. Maurer and J. Zowe. First and second order necessary and sufficient optimality conditions for infinite-dimensional programming problems. Math. Programming, 16(1):98-110, 1979.

[6] M. Hintermüller. A primal-dual active set algorithm for bilaterally control constrained optimal control problems. Quart. Appl. Math., 61(1):131-160, 2003.

[7] M. Hintermüller, K. Ito, and K. Kunisch. The primal-dual active set strategy as a semismooth Newton method. SIAM J. Optim., 13(3):865-888, 2003.

[8] P.G. Ciarlet. The Finite Element Method for Elliptic Problems. North-Holland, Amsterdam, 1978.

[9] CALFEM. A finite element toolbox to MATLAB, Department of Mechanics and Materials, Lund University, Sweden, 2000. http: / / www . byggmek. Ith. se/CALFEM.

[10] S. Klinkel and W. Wagner. A geometrically nonlinear piezoelectric solid shell element based on a mixed multi-field variational formulation. International Journal for Numerical Methods in Engineering, 65(3):349-382, 2006.

[11] L. Costa, P. Oliveira, I.N. Figueiredo, and R. Leal. Actuator effect of a piezoelectric anisotropic plate model. Mechanics of Advanced Materials and Structures, 2006, to appear.

ISABEL NARRA FIGUEIREDO

Center of Mathematics, University of Coimbra, Apartado 3008, 3001-454 Coimbra, Portugal

E-mail address: isabelfamat.uc.pt

URL: http: //www.mat.uc.pt/ isabelf

GEORG STADLER

Center of Mathematics, University of Coimbra, Apartado 3008, 3001-454 Coimbra, Portugal

E-mail address: georgst @mat .uc.pt 